\title{
La quête bouddhiste et l'esthétique postmoderniste russe : le cas de Viktor Pelevin
}

Anastasia de La Fortelle

\section{(2) OpenEdition}

\section{Journals}

Édition électronique

URL : http://journals.openedition.org/edl/787

DOI : $10.4000 /$ edl. 787

ISSN : 2296-5084

Éditeur

Université de Lausanne

\section{Édition imprimée}

Date de publication : 15 septembre 2014

Pagination : $367-378$

ISBN : 978-2-940331-35-2

ISSN : 0014-2026

\section{Référence électronique}

Anastasia de La Fortelle, "La quête bouddhiste et l'esthétique postmoderniste russe : le cas de Viktor Pelevin », Études de lettres [En ligne], 2-3 | 2014, mis en ligne le 15 septembre 2017, consulté le 20 décembre 2020. URL : http://journals.openedition.org/edl/787 ; DOI : https://doi.org/10.4000/edl.787 


\section{LA QUÊTE BOUDDHISTE ET L'ESTHÉTIQUE POSTMODERNISTE RUSSE: LE CAS DE VIKTOR PELEVIN}

Notre article vise à analyser la fonction sémantico-poétique que remplissent les éléments de la philosophie bouddhique dans l'œuvre de l'écrivain russe contemporain Viktor Pelevin, notamment dans son roman La Mitrailleuse d'argile. Le texte retrouvé dans une «Mongolie intérieure» par le «Président du Front Bouddhiste de la Libération totale et définitive" représente un kaléidoscope de réalités virtuelles, hypothétiques, hallucinatoires, qui semblent graviter autour de la notion dominante dans l'imaginaire de Pelevin: celle de la vacuité. L'interrogation sur les origines et les enjeux de cette notion induira la question du sens de la quête bouddhique - pure expérience esthétique ou nouveau refuge idéologique - au sein du postmodernisme russe.

Au début des années 1990, où Viktor Pelevin fait ses débuts en tant qu'écrivain, le paysage littéraire se structure dans un croisement de tensions opposées. Avec la publication massive de textes interdits, la littérature, à un niveau déterminé par des facteurs extra-artistiques (celui que les formalistes qualifient de "genèse»), accomplit une importante mission mnémonique, devient l'un des moyens de retrouver la mémoire culturelle et assure, en conséquence, la continuité historique. Cependant, au niveau qui renvoie à la série intra-littéraire et est déterminé par la nature même de ce qui change (niveau de l'«évolution", selon la terminologie formaliste), domine une tension de rupture ${ }^{1}$. La littérature se lance à la recherche de nouvelles formes et combinaisons, elle devient très linguocentriste, véritable atelier polyglotte; elle joue à la tour de Babel, revendiquant une pluralité de langages tant au niveau de son objet qu'au niveau de la représentation, tant sur le plan littéral que symbolique. Différentes

I. Cf. à ce propos notre article «Zakony literaturnoj èvoljucii». 
tentatives taxonomiques visent à ordonner le foisonnement de stratégies d'écriture tout en indiquant les itinéraires de rupture. Selon plusieurs critiques, des décombres de la littérature soviétique surgissent tout à la fois les postmodernismes romantique, constructiviste, métaphysique, les réalismes magique, ludique, fantastique, le postréalisme, le «métatransréalisme».

Il n'en reste pas moins que derrière la multiplicité de ces "-ismes" apparaît un principe structurant, un antagonisme de deux paradigmes ${ }^{2}$, marquant le retour, comme dans un cycle nouveau, d'un "déjà-vu»: l'ancienne opposition de «l'art pour l'art» et de «l'art comme reflet de la vie». Une tendance à la représentation illusionniste du monde objectal, la priorité et la transparence du signifiant, la présence de l'auteur, une hiérarchie bien définie de valeurs relèvent de la convention que l'on peut qualifier de réaliste. La seconde convention est postmoderne: un espace artistique se crée, qui s'approprie des discours et langages divers, détruit les uns, déconstruit les autres, et revendique l'autoréférentialité du signe artistique. Inachèvement et fragmentation, démesure et éclatement, intertextualité, codages visant une multiplicité d'interprétations, intrigue inexistante, brouillage des contours spatio-temporels du récit, etc. - tous ces mécanismes font partie du paradigme postmoderne auquel est rattaché traditionnellement le nom de Viktor Pelevin, lequel, en outre, est souvent considéré comme l'un de ses représentants phares.

Viktor Pelevin est un écrivain fort énigmatique. En témoigne déjà le fait que pour un grand nombre de lecteurs (prétendant, par ailleurs, être de grands admirateurs des textes peleviniens), la question - Pelevin existe-t-il réellement? - est bien présente. En effet, l'interrogation sur la véritable identité de l'écrivain (une femme, un ordinateur, un groupe littéraire?) traverse les articles de presse. Cette interrogation semble provoquée par le comportement même de l'écrivain qui, consciemment, voire méthodiquement, enveloppe sa vie d'une auréole de mystère. En dépit de son immense popularité auprès d'un lectorat très hétérogène (du point de vue de l'âge et du milieu social) ${ }^{3}$, Pelevin est totalement absent des médias; il ne donne presque jamais d'interviews, ne participe pas aux

2. Cf. à ce sujet, par exemple, M. Berg, Literaturokratija.

3. On peut dire que Pelevin a réalisé sur le sol russe le fameux slogan lancé à l'aube du postmodernisme par un de ses théoriciens américains Leslie Fiedler: "Cross the border - Close the gap" (cf. L. Fiedler, Cross the Border - Close the Gap). 
salons du livre et ne rencontre pas son public; sur ses rares photos, ses yeux sont toujours cachés derrière des lunettes noires.

Dans ce contexte de doute et de mystère engendré par la personne de Pelevin, ses œuvres, au contraire, apportent, semble-t-il, quelques certitudes. Ainsi, une des problématiques clés de Viktor Pelevin depuis ses débuts est celle de la réalité qui apparaît comme une catégorie relative et précaire:

[...] mais n'était-ce pas la seule chose que j'aie toujours été capable de faire: tirer au stylo sur la boule de miroir de ce faux monde? ${ }^{4}$

Certes, le paradigme de la réalité disparue est propre à toute la littérature actuelle ("chaque livre tend aujourd'hui à devenir un reportage fait à partir de l'abîme", remarque A. Genis ${ }^{5}$ ). Or, il est non moins clair que chez Pelevin, beaucoup plus que chez n'importe quel autre écrivain russe contemporain, c'est ce paradigme qui alimente les principales tensions narratives, sémantiques et poétiques du texte. Ainsi, dès les premières œuvres peleviniennes, c'est bien le réel s'anéantissant qui entraîne une remise en cause de la catégorie fictionnelle du personnage. En 1991, le héros du Prince du Gosplan "s'effrite» sous la pression du monde virtuel pour changer définitivement de statut et devenir un personnage de jeux vidéo qu'il est censé programmer. En 1993, dans La Vie des insectes, les personnages vivent simultanément deux incarnations - humains et insectes à la fois. Il ne s'agit pourtant pas d'une métamorphose, puisque le moment même de la transformation, du passage, est absent, mais cette double incarnation se réalise dans le texte, à travers un changement constant de focalisation.

Ce ne sont que deux exemples, néanmoins la logique narrative est toujours la même dans tous les textes de Pelevin: utilisant différents procédés (brouillage des contours spatio-temporels du récit, fragilisation du statut du personnage...), il remet en question la stabilité et l'authenticité de la réalité, s'interroge sur la solidité de la frontière entre le réel et l'imaginaire, le virtuel ${ }^{6}$. La décomposition par divers procédés de la catégorie

4. V. Pelevine, La Mitrailleuse d'argile, p. 367.

5. A. Genis, «Pole čudes», p. 90.

6. De ce point de vue, les textes peleviniens ne peuvent pas être qualifiés de fantastiques dans le sens qu'accorde à ce terme Tsvetan Todorov, pour qui le genre de la littérature fantastique suppose une hésitation permanente de la part du lecteur 
de la présence culmine, dans plusieurs romans peleviniens, dans l'articulation de la notion de «vide». C'est par cette dernière que le paradigme postmoderniste va rejoindre, dans l'œuvre de Pelevin, ce qui pourrait être appelé - avec tout de même une certaine prudence - "une quête bouddhiste».

Pelevin commence à s'intéresser au bouddhisme dès son plus jeune âge, à l'époque soviétique où la littérature religieuse est formellement interdite, mais où abondent, en revanche, les dictionnaires d'athéisme et les brochures méthodologiques destinés aux professeurs d'athéisme scientifique. Toute cette littérature très riche et détaillée est alors facilement accessible dans n'importe quelle bibliothèque. Pelevin évoque ce fait ironiquement, comme si l'information exhaustive sur, par exemple, le taoïsme était nécessaire aux enseignants d'athéisme pour qu'ils soient prêts à combattre une éventuelle épidémie de ce genre quelque part dans la région de Moscou ${ }^{7}$. A l'époque, le bouddhisme apparaît à Pelevin comme la seule religion qui ne ressemble pas à «une projection du pouvoir soviétique sur le domaine de l'esprit». Et, de ce point de vue, il semble être à la fois étrangement "excitant» et "rassurant». Aujourd'hui, Pelevin ne se veut pas un spécialiste du bouddhisme, mais il se positionne comme quelqu'un pour qui le Dharma de Bouddha représente le meilleur moyen d'«entraîner sa conscience».

Les références au bouddhisme s'inscrivent dans les textes peleviniens de différentes manières. Elles peuvent s'insérer de façon indirecte, voire cryptée, exigeant du lecteur un travail de "repérage» et de déchiffrement (résumé fait par la radio du Livre tibétain des morts dans «Les nouvelles du Népal " ${ }^{8}$; évocation, au début de La Mitrailleuse d'argile, de la revue Iside, principale publication des martinistes russes du début du $\mathrm{XX}^{\mathrm{e}}$ siècle $^{9}$ ); elles apparaissent également sous une forme volontairement camouflée, comme dans La Vie des insectes, où une libellule chante

doutant de la vraie nature des événements décrits: réelle ou surnaturelle (cf. T. Todorov, Introduction à la littérature fantastique).

7. Cf. à ce propos l'interview de Pelevin: <http://pelevin.nov.ru/interview/obomb/r.html>.

8. A. Genis, «Pole čudes», p. 89.

9. Cf. les nombreuses références bouddhistes dans la préface d'A. Trojanovskij à Y. Ramacharaka, Fourteen Lessons in Yogi Philosophy and Oriental Occultism, publié en 1913 (<http://scriptures.ru/yoga/osnovy_miy.htm>) sous le titre de Osnovy mirosozercanija indijskix bogov. 
à la télé: «Завтра улечу / В солнечное лето / Будду делать все / Чmo захочу " ["Demain, je volerai / Dans l'été ensoleillé / Et ferai ce que bouddrai $\left.{ }^{10}\right]$. En russe, l'accusatif de Bouddha et la première personne du verbe "être" au futur se prononcent de la même manière, mais diffèrent dans l'écriture par la présence de deux «d" pour Bouddha et d'un seul pour la forme verbale. Ainsi, la référence au bouddhisme s'incruste-t-elle, dissimulée sous une faute d'impression (deux « $\mathrm{d}$ » au lieu d'un seul), accordant finalement au texte de Pelevin un sens sacré (la transformation d'une phalène en luciole en tant quallégorie de l'Eveil et de l'Illumination bouddhistes ${ }^{11}$ ).

Dans d'autres textes peleviniens, les références bouddhistes sont, au contraire, explicites. Dans Homo Zapiens, le bouddhisme apparaît constamment comme une sorte de modèle paradigmatique qui sert à mieux cerner les contours de la réalité postsoviétique où la publicité acquiert le caractère d'un rituel magique. Indépendamment de ce que cette superposition de deux "plans" sacrés peut tenir d'un jeu postmoderniste éclectique, satirique et parodique, le lecteur de Homo Zapiens découvre, d'un point de vue purement formel, dans la philosophie bouddhiste ce fond narratif continu sur lequel s'opère la production de sens dans le contexte de la civilisation de la télé. Ainsi les résultats obtenus par la technologie publicitaire «zombifiant» le spectateur à l'aide d'une savante application de différents mécanismes de manipulation, sont-ils exprimés à travers la référence à ce même paradigme bouddhiste. La publicité apparaît comme un terrain postmoderne efficace de réincarnation :

D'un point de vue bouddhiste, le sens de la publicité est très simple: elle essaie de convaincre sa cible que la consommation d'un produit qu'elle vante conduit à une nouvelle incarnation, plus élevée et bénéfique, non pas après la mort, mais tout de suite après l'acte de consommation ${ }^{12}$.

L'œuvre pelevinienne dans laquelle le message bouddhiste d'un fond narratif se transforme en noyau thématique central structurant le texte à tous ces niveaux, est le roman publié en russe sous le titre de Tchapaïev i Pustota et en français sous celui de La Mitrailleuse d'argile. Au centre

Io. V. Pelevine, La Vie des insectes, p. 187.

II. Cf. à ce sujet A. Genis, "Pole čudes», p. 88.

I2. V. Pelevine, Homo Zapiens, p. 167. 
de ce texte, découvert (comme le lecteur l'apprend dès le début) au sein d'une «Mongolie intérieure» par le "Président du Front Bouddhiste de la Libération totale et définitive», se trouve le personnage de Vassili Tchapaïev, légendaire commandant de l'Armée rouge, héros de la Guerre civile (1917-1923). Le second héros est le non moins célèbre aide de camp historique de Tchapaïev, Petr Issaev, qui apparaît dans le roman sous l'identité du poète pétersbourgeois Petr Pustota (son nom désignant en russe le «vide»). Ces figures historiques de la période révolutionnaire sont devenues, au cours du $\mathrm{XX}^{\mathrm{e}}$ siècle, les personnages récurrents de nombreuses plaisanteries regroupées dans une sorte de cycle folklorique «Tchapaïev et Petka». Or, Pelevin détruit ou plutôt déconstruit, au sens postmoderniste du terme, la réalité historique en la passant à travers le filtre de la philosophie bouddhiste: le héros légendaire de l'Armée rouge Vassili Tchapaïev (et l'on retrouve chez Pelevin certaines caractéristiques du personnage historique, évoquées, par exemple, dans des textes du réalisme socialiste) explique à plusieurs reprises à son aide de camp Petr que les phénomènes extérieurs et intérieurs, physiques et psychiques n'ont pas d'existence autonome, mais qu'ils ne sont que des étiquettes illusoires produites par la conscience. Cette vision de Tchapaïev fait écho à celle de l'auteur lui-même qui semble être surtout attiré par les inspirations bouddhistes du Madhyamaka et du Mahāyāna et cite l'Avatamsakasütra sous un angle bien précis:

Que veut dire s'envoler vers d'autres univers? Tous les univers sont ici, là où l'on se trouve. Pour y accéder, point n'est besoin de s'envoler où que ce soit. Le texte bouddhiste du "Sutra Avatamsaka» dit que le monde se crée exclusivement dans l'esprit. Chaque déplacement d'un espace vers un autre est avant tout un acte psychique ${ }^{13}$.

Il est clair que Pelevin en ne privilégiant qu'un aspect de la cosmologie bouddhiste très complexe, voire - pour le non-spécialiste - contradictoire, instrumentalise la sagesse orientale afin d'explorer une problématique qu'il juge centrale pour son œuvre: celle de la conscience humaine ${ }^{14}$. C'est dans cette perspective qu'il faut interpréter maintes

I3. Cf. <http://pelevinlive.ru/14>.

I4. Cf. à ce propos l'interview de Pelevin dans laquelle le terme de "conscience» est abondamment utilisé pour désigner la charge sémantico-thématique centrale de ses textes (<http://pelevin.nov.ru/interview/o-bomb/1.html>). 
citations cryptées de la tradition prajñāpāramitā qui parsèment le texte de La Mitrailleuse d'argile: "Toute forme, c'est le vide [...] le vide prend n'importe quelle forme ${ }^{15}$. La signification ultime qu'accorde Pelevin à ces références ne correspond pas à l'exégèse traditionnelle de la "vérité absolue» du texte bouddhiste. La vacuité apparaît, chez Pelevin, en tant qu'absence de toute essence en soi, stable, durable et inchangeable, non pas parce qu'elle renvoie à l'interdépendance absolue de tous les phénomènes et, donc, à l'inexistence de l'existence intrinsèque, mais avant tout parce qu'elle désincarne la réalité phénoménale en la plaçant à l'intérieur d'une conscience ( $«-$ Et où est l'Univers? [...] - Il est en soi. - Et où en soi ? - Dans ma conscience» $\left.{ }^{16}\right)$ :

- Petka! appela Tchapaïev. Où es-tu?

- Nulle part! marmonnai-je en guise de réponse.

- Ça alors! hurla Tchapaïev subitement. Bravo! Demain je te féliciterai devant les troupes. Tu comprends donc tout! [...].

- Tout ce que nous voyons, Petka, se trouve dans notre conscience. Voilà pourquoi il est impossible de dire que notre conscience se trouve quelque part. Nous ne nous trouvons nulle part pour la simple raison qu'il n'existe aucun lieu dont on pourrait dire que nous sommes à l'intérieur ${ }^{17}$.

Il faut noter que ces motifs de l'effritement de la réalité phénoménale et de la fuite vers le vide, ont été souvent interprétés de l'intérieur d'une perspective postmoderniste plus générale - celle de la sortie de l'histoire qui n'apparaît plus comme cohérente, mais est soumise au hasard, a un caractère paralogique. De même, dans le vide pelevinien - présent sous différentes incarnations dans tous les textes de l'écrivain - on perçoit fréquemment une réplique à la théorie des simulacres et de la simulation de Jean Baudrillard qui conçoit l'époque postmoderniste comme une ère de simulation (s'opposant à la représentation) et de simulacres (s'opposant à l'image

15. V. Pelevine, La Mitrailleuse d'argile, p. 339. Cf. «Le cœur de la perfection de sagesse", in G. Driessens (tr.), La Perfection de sagesse, p. 147: "La forme est vide, la vacuité est la forme. La vacuité n'est pas autre que la forme, la forme n'est pas autre que vacuité. De même, la sensation, la notion, la formation et la conscience sont vides. Ainsi [...] tous les phénomènes sont vacuité, sans caractéristiques, sans naissance, sans arrêt, sans impuretés, sans séparation d'avec les impuretés, sans diminution ni accroissement. »

I6. V. Pelevin, Čapaev i Pustota, p. 152.

I7. V. Pelevine, La Mitrailleuse d'argile, p. 165. 
mimétique ou transgressive) où le réel cède la place à l'hyper-réel et la carte de l'Empire se substitue au territoire lui-même ${ }^{18}$. Le fait qu'il n'y ait pas d'espoir pour le sens, la déperdition de ce dernier sont directement liés chez Baudrillard à l'action "dissolvante, dissuasive, de l'information, des media et des mass-media " ${ }^{19}$. Pelevin, en effet, réplique à Baudrillard dans Homo Zapiens où tous les modes d'expression sont engloutis par le phénomène que le philosophe français appelle la publicité absolue se confondant avec elle-même et "dont l'érotisme n'est que l'index auto-érotique d'un système qui ne fait plus que se désigner lui-même... " ${ }^{20}$. En écho à un autre livre de Baudrillard, La Guerre du Golfe n'a pas eu lieu, Pelevin ${ }^{21}$ fait découvrir à son héros, jeune creator de spots publicitaires, que tous les hommes politiques, ainsi que tous les événements sociaux ne sont en réalité que des coquilles vides, des créations virtuelles engendrées, manipulées et contrôlées par la télévision. A la fin de ce roman, où les objets se trouvent régulièrement remplacés par les signes de leur existence (les œuvres d'art par les quittances d'achat, par exemple), le héros rejoint littéralement ce monde du néant et du simulacre en se laissant numériser en image 3D.

Le vide exploré par Pelevin dans le roman La Mitrailleuse d'argile qui, par ailleurs, recourt à plusieurs procédés de l'écriture postmoderniste (la mise à nu du caractère paralogique de l'histoire, par exemple) n'est pourtant point corrélatif de celui véhiculé par la notion de simulacre. Vassili Tchapaïev, qui est une réincarnation du Bouddha Anagama, un gardien du Dharma, initie son élève Pustota à l'école de la vacuité afin de le mener tout droit à l'Eveil et l'Illumination. Le parcours initiatique du héros se réalise grâce à la pratique des koans zen (courtes phrases et série de phrases énigmatiques et/ou paradoxales remettant en cause et transgressant la logique ordinaire de raisonnement). La concentration et le contrôle du mental exigés par les koans en tant qu'objets de méditation, tout en favorisant le travail de discernement (vérité/ égarement), permettent au héros de se rapprocher progressivement de la libération absolue, de cette Mongolie intérieure qui ne «se trouve pas à l'intérieur de la Mongolie, mais à l'intérieur de celui qui voit le vide»:

I8. J. Baudrillard, Simulacres et simulation, p. 10.

I9. Ibid., p. 120.

2o. Ibid., p. 135.

2I. Pelevin par ailleurs, affirme lui-même dans une interview: «Du point de vue phénoménologique, tout homme politique n'est qu'une émission de télé.» (<http:// pelevin.nov.ru/interview/o-bomb/1.html>). 
- Kotovski a compris qu'il n'y avait pas de forme. Mais il n'a pas compris qu'il n'y avait pas de cire non plus.

- Et pourquoi n'y en avait-il pas?

- Mais parce que, Petka, écoute-moi attentivement, la cire et le tordboyaux peuvent prendre n'importe quelle forme, mais ne sont euxmêmes que des formes.

- Les formes de quoi?

- C'est ça, le truc. Ce sont des formes dont on peut seulement dire qu'il n'y a rien qui les prend. Comprends-tu? C'est pourquoi, en vérité, il n'y a ni cire ni tord-boyaux. Il n'y a rien. Et même cette négation n'existe pas ${ }^{22}$.

Cet enracinement dans un nulle part, dans un vide - thème préféré des koans de Tchapaïev - est, d'ailleurs, mise en abîme par la structure du roman tout entier, puisque les chapitres où Petr se trouve lié à Tchapaïev en 1919 alternent avec ceux où ce même Petr Pustota est un patient d'un hôpital psychiatrique moscovite dans les années 1990. Chaque Petr rêve de l'autre (il s'agit plutôt de cauchemars), mais ni le lecteur ni le héros lui-même ne sauront jamais quel Pustota est authentique. Savoir, au demeurant, inutile: comme l'explique une fois de plus Tchapaïev, la question de la frontière entre rêve et réalité n'a pas le droit d'exister, puisque elle-même, n'existe pas:

Ah! Petka, Petka, soupira Tchapaïev. J'ai connu un communiste chinois nommé Tseu Zhuang. Dans ses songes, il se voyait comme un papillon rouge qui voletait dans l'herbe. Et lorsqu'il se réveillait, il ne parvenait pas à comprendre s'il était un papillon qui rêvait qu'il faisait de l'action clandestine, ou un révolutionnaire qui planait de fleur en fleur. Un jour en Mongolie, on arrêta ce Tseu Zhuang pour sabotage. Au cours de son interrogatoire, il dit qu'il était en réalité un papillon qui voyait tout cela dans un rêve. Comme c'était le baron Jungern qui l'interrogeait et que c'est un homme très clairvoyant, il lui demanda pourquoi ce papillon travaillait pour les communistes. Tseu répondit qu'il n'était pas pour les communistes. Alors pourquoi menait-il des activités subversives? Il répondit que toutes les actions humaines étaient tellement laides que cela n'avait pas la moindre importance de savoir de quel côté on se trouvait. - Et après? Que s'est-il passé? -

22. V. Pelevine, La Mitrailleuse d'argile, p. 327. 
Rien. Ils l'ont collé contre un mur et réveillé. - Et lui? - Tchapaïev haussa les épaules. - Je suppose qu'il a continué son vol ${ }^{23}$.

L'insistance sur le réel qui n'existe que dans la mesure où il apparaît comme sa propre absence, côtoie dans le roman une autre vision non moins insistante: la sortie du samsara existe (comme existe un moyen de quitter l'asile des fous), l'accès au vide flamboyant qu'est le nirvana (dans le texte - la Mongolie intérieure et l'Oural), lui, est bien réel. Il l'est aussi bien pour Pustota (transgressant, à la fin du roman, l'arbitraire du signe et «fusionnant» avec son nom), que pour d'autres patients de l'hôpital psychiatrique qui, par une thérapie de groupe, peuvent accéder, dans un mouvement cathartique, à une libération:

Sans réfléchir une seconde de plus, je bondis sur mes pieds, pris de l'élan et me jetai dans l'Oural. [...] Je vis l'endroit où le flot commençait et je compris aussitôt que c'était là ma véritable maison ${ }^{24}$.

Ainsi, il semblerait que, dans La Mitrailleuse d'argile, il s'agisse, non de relater comment la réalité se transforme en simulacre, mais de s'interroger sur la naissance de la réalité à partir d'un vide ${ }^{25}$. De ce point de vue, on pourrait émettre l'hypothèse que Pelevin, à travers sa quête bouddhiste, persiste à poursuivre là où Jean Baudrillard s'est arrêté dans son discours sur la postmodernité comme ère de la simulation:

L'imaginaire était l'alibi du réel, dans un monde dominé par le principe de réalité. Aujourd'hui, c'est le réel qui est devenu l'alibi du modèle, dans un univers régi par le principe de simulation. Et c'est paradoxalement le réel qui est devenu notre véritable utopie - mais une utopie qui n'est plus de l'ordre du possible, celle dont on ne peut plus que rêver comme d'un objet perdu ${ }^{26}$.

Anastasia DE LA FORTELLE

Université de Lausanne

\footnotetext{
23. Ibid., p. 229.

24. Ibid., p. 341.

25. Cf. à ce propos M. Lipoveckij, Paralogii. Transformacii (post)modernistskogo diskursa $v$ russkoj kul'ture 1920-2000-x godov.

26. J. Baudrillard, Simulacres et simulation, p. 179.
} 


\section{BIBLIOGRAPHIE}

\section{Textes}

Driessens, Georges (tr.), La Perfection de sagesse: Soutras courts du Grand Véhicule suivis de L'enseignement d'Akshayamati, Paris, Seuil, 1996. Pelevine, Viktor, La Vie des insectes, Paris, Seuil, 1995.

Pelevine, Viktor, La Mitrailleuse d'argile, Paris, Seuil, 1996.

Pelevin, Viktor, Čapaev i Pustota, Moskva, Vagrius, 2000.

Pelevine, Viktor, Homo Zapiens, Paris, Seuil, 2001.

\section{Etudes}

Baudrillard, Jean, Simulacres et simulation, Paris, Galilée, 1981.

—, La Guerre du Golfe n'a pas eu lieu, Paris, Galilée, 1991.

Berg, Mihail, Literaturokratija, Moskva, NLO, 2000.

DE LA Fortelle, Anastasia, "Zakony literaturnoj èvoljucii: K voprosu izučenija novejšej russkoj literatury", in Mirgorod, $n^{\circ}$ III, Akademia Podlaska/UNIL, Siedlce (à paraître en 2014).

Fiedler, Leslie, Cross the Border - Close the Gap, New York, Stein \& Day, 1972.

Genis, Aleksandr, "Pole čudes», in Ivan Petrovič umer. Stat'i i rassledovanija, Moskva, NLO, 1999.

Lipoveckij, Marc, Paralogii. Transformacii (post)modernistskogo diskursa $v$ russkoj kul'ture 1920-2000-x godov, Moskva, NLO, 2008.

Todorov, Tzvetan, Introduction à la littérature fantastique, Paris, Seuil, 1970. 
